\title{
Neural Networks and Regional Science Modeling: A Survey of Techniques for Complex Spatial Analysis
}

\author{
Thomas G. Wier and Vir V. Phoha*
}

\begin{abstract}
This study examines the value of utilizing neural net modeling for issues relating to optimization across a network of cities in space. Neural nets are made up of many nonlinear computational elements that operate in parallel and are arranged in a manner similar to biological neural nets. Defining a neural net model involves specifying a net topology, arrangement of nodes, training or learning rules, adjustment of weights associated with connections, node characteristics, and rules of transformation from input to output. All of these are the major issues in such regional problems as labor force migration and firm location.
\end{abstract}

\section{INTRODUCTION}

The purpose of this paper is to shed light on the value of utilizing existing neural network modeling techniques for issues relating to optimization and classification across a network of city-centric economic markets in space. This could be the optimization of costs by a firm in a location decision or of income by a worker. Classification models could be used to simulate the necessary labor force migration into regionalized locations or "classifications" of employment opportunities at actual firm locations to minimize structural unemployment. Neural nets are made up of many nonlinear computational elements that operate in parallel and are arranged in a manner similar to biological neural nets. Defining a neural net model involves specifying a net topology, such as an interstate highway system; arrangement of nodes, such as the geographic arrangement of cities; training or learning rules, such as microeconomic decision theory or macroeconomic policy making; adjustment of weights associated with connections, such as location decision criteria; node characteristics; and rules of transformation from input to output. All of these are major issues in fundamental regional science problems, such as labor force migration, firm location, and structural unemployment.

A major advantage of neural nets is the ability to examine many competing hypotheses at the same time. Neural nets are more robust than statistical techniques when underlying distributions are generated by nonlinear processes and are strongly non-Gaussian, as is the case in many economic systems. Neural nets are also nonparametric and make weak assumptions about the underlying distribution of data as compared to standard statistical techniques.

Neural networks have been successfully applied across several fields, including economics of financial markets, decision science, image processing, and

\footnotetext{
"Department of Economics, Northeastern State University, Tahlequah, OK; and Department of Computer Sci-
} ence, Louisiana Tech University, Ruston, LA. 
particularly to optimization problems such as the traveling salesman problem (Hopfield and Tank 1985). The improvements made are of an order of magnitude over conventional algorithms. Neural networks work in a parallel and distributed fashion and hence have relatively reduced computational times, which allow more flexibility of experimentation.

This paper will be an examination of these eight and their potential for applications in regional economics:

Unsupervised Learning: (1) Hebb Type Learning, (2) Competitive Learning, (3) Kohonen's Algorithm, and (4) Carpenter Grossberg Classifier.

Supervised Learning: (1) Multilayer Perceptron, (2) Hopfield Model, (3) Hamming Net, and (4) Boltzman Machines.

\section{TRADITIONAL RESEARCH APPROACHES}

Conventional techniques for modeling labor force migration and firm location have been limited by both the technique of analysis and the availability of data.

\section{Labor Force Migration}

Traditionally, data has been collected periodically on each place by the Census Bureau and statistical techniques that compare two points at a time have been utilized. These are referred to as "Origin-Destination" models (Greenwood 1969; Kau and Sirmans 1977; Herzog and Schlottman 1981, 1982, 1995; Levy and Wadycki 1973). This work has mainly focused on verification of the human capital framework as an acceptable model for migration. The human capital model assumes at its foundation that the discounted value of earnings at place " $j$ " outweigh the discounted value of earnings at place " $i$ " plus the relocation cost from " $\mathrm{i}$ " to " $\mathrm{j}$." These models were extended to include origin and destination place characteristics (earnings, employment, quality of life, etc.), characteristics of migrants (personal characteristics such as education, marital status, etc., with number of past moves emerging as a critical factor in future propensity to migrate), and variables representing the difficulty of the journey (mainly distance from " $i$ " to " $j$ "). A regression of likelihood for migration based on specific human capital variables in the origin and destination places results in partial equilibrium weights for each of these determinants. These studies focused on the migration from one specific point to another across a time interval when data was left uncollected. Thus, the usefulness and realism of the analysis is limited by its relative nonspatiality, the pair-wise approach, the lack of dynamic interaction, and the distance between time periods of data collection. Because of the geographic limitations of this type of data, some of the foundational studies have relied on state-tostate migration only (particularly Greenwood 1969). This microeconomic work was, however, successful in demonstrating that there are certain characteristics of places that cause migration. The more recent literature (Herzog, Schlottman, and 
Boehm 1993) examines migration as spatial job search, which is a move in the right direction, but without a comprehensive computational approach, the results are still quite limited.

\section{Firm Location}

Likewise, the literature on the theory of interregional firm location, as opposed to the analysis of competitive locational interdependence within a monocentric city (Hoover 1948; Hotelling 1970; and others), has also focused on partial equilibrium verification of the cost-centered theoretical framework (Weber 1928; Isard 1956; Smith 1971, ch. 8; Moses 1970; Alonso 1969; and many others). The early literature focuses on the location decision as an aspect of the theory of the firm. Total profit was perceived as total revenue minus the sum of total production costs and total transportation costs. The limiting assumptions of perfect competition allowed theorists to also assume that total revenue and total production costs are fixed across space, thus positioning transportation costs as the sole consideration in the location of the firm. Not the least among these assumptions is that all factors of production, like labor and capital (including plants) are both perfectly mobile and informed. The majority of the theories also assume a "uniform transportation network;" that is, a featureless plain with inputs and output markets available at fixed known locations with no fixed path between them. These classical theories, along with the "central place" theories, serve more as conceptualizations of how economic activity and transportation routes are originally organized, rather than as a model of the type of dynamic firm location and relocation needed today.

Later work did attempt to analyze how "fixed path" transportation routes would impact firm location (particularly Palander 1935). These models carried forward all of the restrictive assumptions of those previous, including the often, interregionally, unrealistic assumption that the firm is limited to a single possible source for each of its input and output markets. This is a critical concern and must be expanded here in some detail.

Weber's (1928) original two-dimensional location decision model was optimized with the following formula:

$$
\min \sum_{\mathrm{i}=1}^{\mathrm{n}} \mathrm{IW}_{\mathrm{i}} \mathrm{d}_{\mathrm{i}},
$$

where IW is the ideal weight of the input at place " $\mathrm{i}$ " and " $\mathrm{d}$ " is the distance that input must be shipped. The ideal weight is the product of the transportation rate for the input and a technical coefficient that represents the weight of the input per unit of product weight. In Palander's (1935) analysis of firm location with a transportation network, this results in a basic decision rule: if you have a location with a dominant ideal weight, no matter what the network is, the plant will be located there. A dominant ideal weight is one that is greater than the sum of the ideal weights in all other locations. If you do not have a dominant weight, then the location is based upon the arrangement of weights within the network. The theory 
then demonstrates the location decisions that would occur given different weights and network types, such as linear, forked, circular, and looped arrangements.

The key in this analysis is not the validity of modeling location in this manner, but the validity of its assumptions. This model of location decision making assumes that the firm is limited to specific input sources without other locations competing for the opportunity to supply the materials and labor inputs. That is, that there is no interregional competition for firm location. If the firm is limited to one market region with inputs in a single set of fixed locations, then this model is sufficient for such decision making. If, however, the firm is faced with a choice of markets in which to locate with each having the necessary inputs, such as firms whose key input is labor, then this model is insufficient for decision making. These models also continue to assume that the dominant issue in the firm's location decision is the transportation cost of inputs.

In separate works, Chinitz (1969) and Barloon (1969) concluded that the input orientation of firms caused by the advancement of the industrial revolution changed direction around 1929. An ever-increasing shift toward market location has dominated since (they do not, however, agree on whether the changes in transportation and technology caused the change in industry or vice versa). The key issues in this change were:

1.Transportation rates have risen faster than wholesale prices;

2.Freight rates on raw materials are generally lower than rates for finished goods on a pound for pound basis;

3.This gap has been widening over time and technological improvements have more greatly reduced the rates of raw materials transport;

4. Technological change has also reduced terminal costs more than linehaul costs;

5.The cost of short hauls has been reduced more than long ones; and

6. The development of trucking has also favored the large shipper.

Given this information, an extremely important issue is revealed: which market from among the various available choices will be selected and why? This question remains unanswered because this reality remains unmodeled.

\section{Implications}

What these traditions fail to do is demonstrate how dynamic migration or firm location actually takes place given all of the place choices and what the true micro and macroeconomic impacts are in real space and time. That is, how attractive and repulsive variables are impacted over time as migration occurs or, perhaps more importantly, does not occur, and how this affects the overall functioning of the U.S. economy. To state a more complex key question at issue when these two problems are brought to light at the same time, especially where structural unemployment is concerned, how does the interregional relocation of firms impact the migration of labor and vice versa? 


\section{A NEURAL NETWORK APPROACH TO REGIONAL SCIENCE}

The map in Figure 1 is a representation of the BEA Economic Areas. If each numbered market area is conceived as a potential departure point and/or destination for migration or firm location (henceforth referred to as a "node"), then the map can serve as a powerful visual aid. These Economic Areas define the citycentric regional geographic markets within the United States connected by the actual interstate transportation system. The modeler may begin with realistically arranged matrices of the data provided by the BEA, BLS, and other agencies for these Economic Areas. The realism will come from an arrangement of nodes that relate to one another in space in accordance with their relative location in the interstate transportation network. This will allow for the maximum benefit from the particular neural network modeling techniques chosen for application to regional economics from the list of potential architectures discussed below.

FIGURE 1

BEA Economic Areas

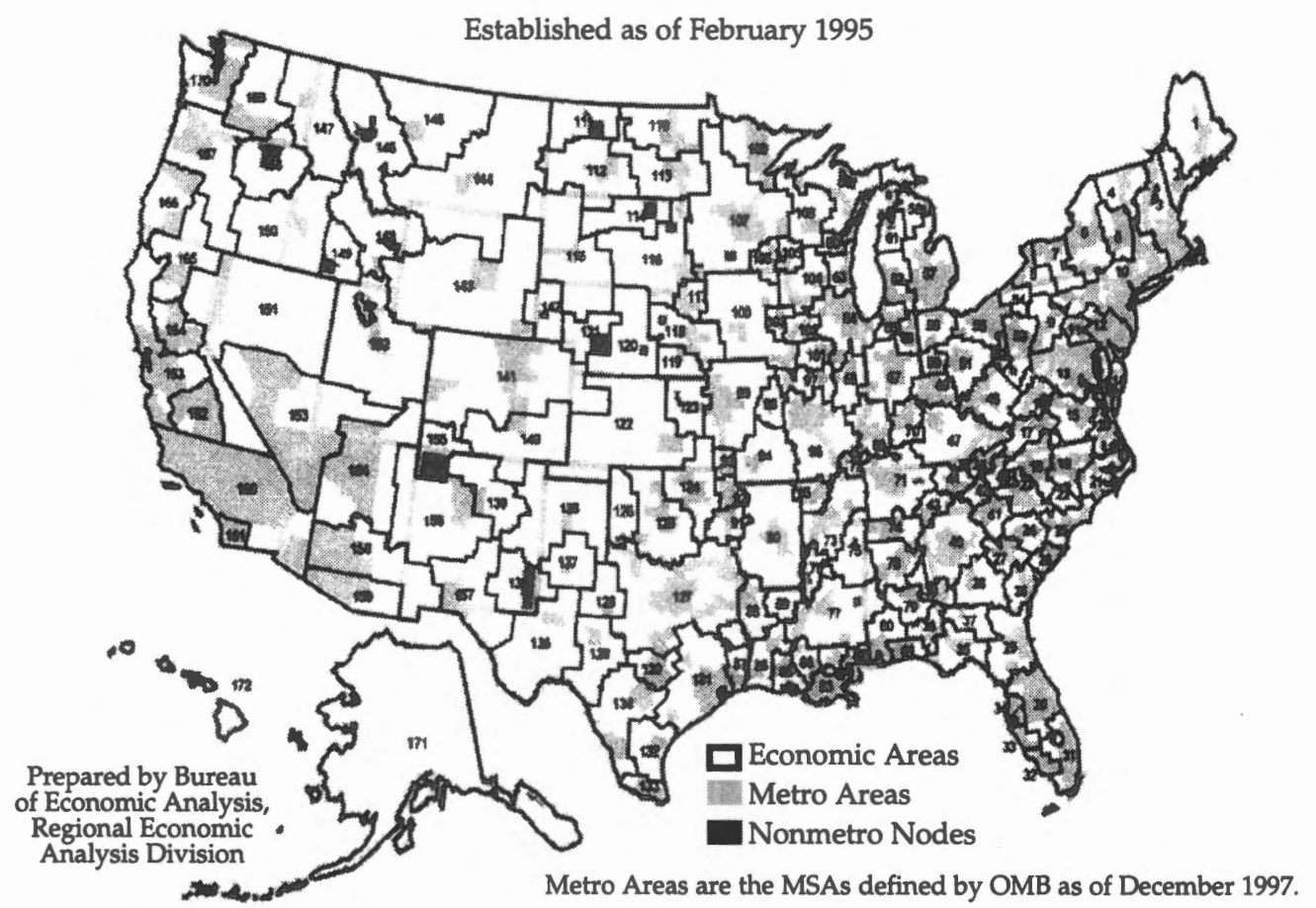

IV. NEURAL NET MODELS

\section{Hebb Type Learning}

Donald Hebb (1949) first proposed a learning scheme for updating node connections that we now refer to as the Hebbian learning rule. He stated that information can be stored in connections, and postulated the learning technique that has made fundamental contributions to neural network theory. According to 
this rule (as formulated in Zurada 1992), the learning signal is equal simply to the neuron's output " $r$ " (see Figure 2 and Equation 2).

FIGURE 2

Hebb Learning

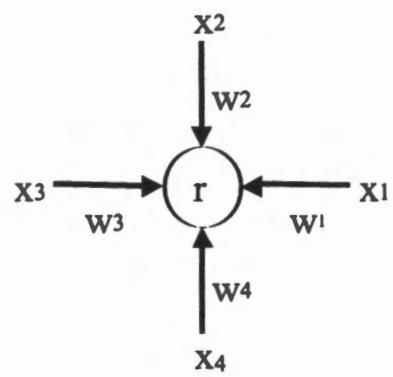

In this rule:

(2)

$$
r=f\left(w_{i}^{t} x\right) \text {, }
$$

where $f$ is a nonlinear function, $w_{i}$ is a weight vector of component $w_{i j}$ connections of the jth input with the ith node, and $x$ is the input vector. The increment $\Delta w_{i}$ of the weight vector becomes:

$$
\Delta w_{i}=\eta f\left(w_{i}^{t} x\right) x,
$$

where $\eta$ is a positive number called the learning constant and determines the rate of learning.

"Learning" is the adjustment of the strength of the weight or connection between the input and the node. The rule is an implementation of the fundamental neuro-biological statement: "When an axon of a cell A is near enough to excite a cell $B$ and repeatedly and persistently takes place in firing it, some growth process or metabolic change takes place in one or both cells such that $A^{\prime}$ s efficiency as one of the cells firing B is increased (Hebb 1949, p. 11)." Thus, this concept of strengthening or "learning" forms the foundation of all of the following neural network models. Learning is seen as the strengthening of a connection between nodes that intensifies their relation.

It is a fundamental thesis of the authors that, in regional science terms, this "learning" is what is referred to as "development." In any of the following models, the central place nodes are analogous to the cell and "strengthening" the node can take the form of an increase in labor force or firms. The resulting impact in the node could be decreasing unemployment and equilibration of income or production (or any other spatially organized phenomena a researcher would wish to model).

Each application will involve a process of defining the "learning rate" by adjustment of one or more parameters in order to perfect the fit of the model. In a migration simulation, this would involve examining the correlation between the estimated outputs of a series of iterations to the empirical data in order to "tune" the model. Once tuned, predictive impact application is possible. In the area of 
unemployment analysis, this approach could be taken with a model tuned to migration data in order to simulate the impact of large layoffs such as those recently experienced at Boeing Aircraft. Thus, a properly tuned optimization model could estimate the number of iterations or "years" necessary to redistribute the unemployed and return the system to equilibrium. If the model was set up as a classification algorithm, a neural network could also be used to predict the likely destinations of the migrants.

All of the models presented below have potential for such applications in regional science. The key is to discover which structure relates properly with the particular problem. Also, each approach to the "learning" or update rules used to adjust the connection weights described below must be examined and, if chosen to model a particular phenomenon, developed to reflect accurately real world microeconomic decision making and macroeconomic impact. Thus, a fundamentally mesoeconomic approach is formed.

The Hebbian learning rule represents a purely feedforward, unsupervised learning. Feedforward means that the flow of information moves forward only through the network node, such that the output of a layer is the input to the following layer. There are no explicit feedback connections in feedforward networks. Unsupervised learning assumes that the desired response is not known; thus, explicit error information cannot be used to improve network behavior.

\section{Unsupervised Learning}

The following models fundamentally rely on the same neuro-biological and methodological foundations as the Hebb-type learning discussed above. In unsupervised learning, it is assumed that for each instant of time when the input is applied the desired output is not known; thus, explicit error information is not used to improve network behavior. This implies the same conditions inherent in systems with uncertainty.

\section{Competitive Learning}

In the simplest competitive learning networks (Phoha and Oldham 1996a, b), there is a single layer of output units $\mathrm{O}_{\mathrm{i}}$, each of which is fully connected to a set of inputs $x_{i}$ via connection weights $w_{i j} \geq 0$. The input-to-output relationship can be one of time where the input is the value of the regional economic variable under study in an area at one time period. The output is the value of that same variable in the next time period. The result is a model that demonstrates changes across space over time. A description of the algorithm follows.

Let $x$ be an input to a network of two layers with an associated set of weights $\mathrm{w}_{\mathrm{ij}}$. The standard competitive learning rule (Hertz, Krogh, and Palmer $1991)$ is given by:

$$
\Delta w_{i^{*} j}=\eta\left(x_{j}-w_{i^{*} j}\right),
$$

which moves $\mathrm{w}_{\mathrm{i}^{*}}$ towards $\mathrm{x}$; the $\mathrm{i}^{*}$ implies that only the set of weights corresponding to the winning nodes is updated. The winning node is taken to be the one with the largest output. Another way to write this is: 


$$
\Delta w_{i j}=\eta O_{i}\left(x_{i j}-w_{i j}\right),
$$

where $O_{i}=1$ for $i$ corresponding to the largest output, 0 otherwise. This has the appearance of $\mathrm{Hebb}$ rule with decay. This is the adaptive approach taken by Kohonen (Hertz, Krogh, and Palmer 1991) in his first algorithm (see Kohonen model below). The usual definition of competitive learning requires a winnertake-all strategy. In many cases, this requirement is relaxed to update all of the weights in proportion to some criterion. This form of competitive learning is referred to as leaky learning (see Hertz, Krogh, and Palmer 1991). Hertz, Krogh and Palmer (1991) discuss various forms of this adaptive processing for different problems, including the traveling salesman problem. It has become a standard practice to refer to all of these as Kohonen-like algorithms.

Kohonen's Algorithm

Kohonen's Algorithm adjusts weights from common input nodes to $\mathrm{N}$ output nodes arranged in a two-dimensional grid (see Figure 3 ) to form a vector quantizer. Input vectors are presented sequentially in time and after enough input vectors have been presented, weights specify clusters or vector centers. These clusters or vector centers sample the input space such that the point density function of the vector centers approximate the probability density functions of the input vectors. This algorithm also organizes weights such that topologically close nodes are sensitive to physically similar inputs. Output nodes are thus ordered in a natural fashion. Thus, this algorithm forms feature maps of inputs. A description of this algorithm follows.

FIGURE 3

Kohonen's Algorithm

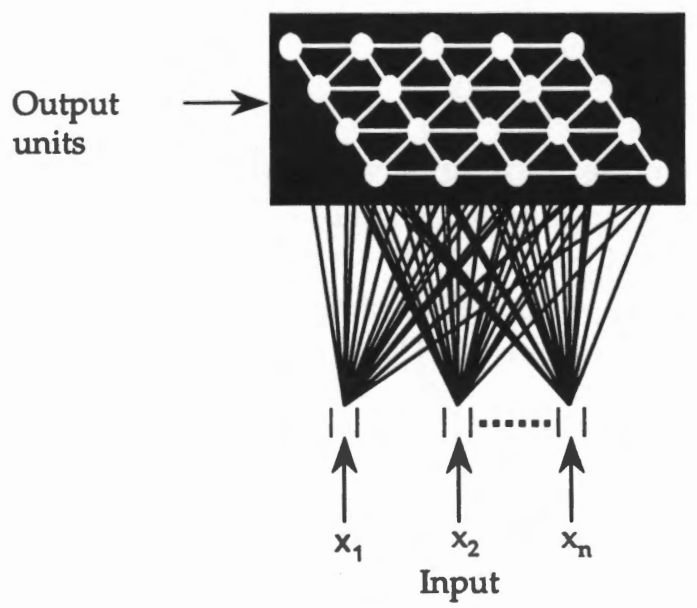

Let $x_{1}, x_{2}, \ldots, x_{N}$ be a set of input vectors, which defines a point in Ndimensional space. The output units $\mathrm{O}_{i}$ are arranged in an array and are fully connected to input via the weights $w_{\mathrm{ij}}$. A competitive learning rule is used to choose a winner unit $i^{*}$, such that 
(6) $\left|w_{i^{*}}-x\right| \leq\left|w_{i}-x\right|$ for all $i$,

then the Kohonen's rule is given by:

$$
\Delta w_{i}=\eta h\left(i, i^{*}\right)\left(x-w_{i}^{\text {Old }}\right) .
$$

Here $h\left(i, i^{*}\right)$ is the neighborhood function such that $h\left(i, i^{*}\right)=1$ if $i=i^{*}$ but falls off with distance $\left|r_{i}-r_{i^{*}}\right|$ between units $i$ and $i^{*}$ in the output array. The winner and close by units are updated appreciably more than those further away. A typical choice for $\mathrm{h}\left(\mathrm{i}, \mathrm{i}^{*}\right)$ is:

$$
\mathrm{e}^{\left.-\left(\mid \mathrm{r}_{\mathrm{i}}-\mathrm{r}_{\mathrm{i}}\right) / 2 \sigma^{2}\right)},
$$

where $\sigma$ is a parameter that is gradually decreased to contract the neighborhood. $\eta$ is decreased to ensure convergence.

\section{Carpenter Grossberg Classifier}

Carpenter and Grossberg (1988), based on their adaptive resonance theory, have developed a net that forms clusters and is trained without supervision. An example of this net with three input and two output nodes is given in Figure 4.

FIGURE 4

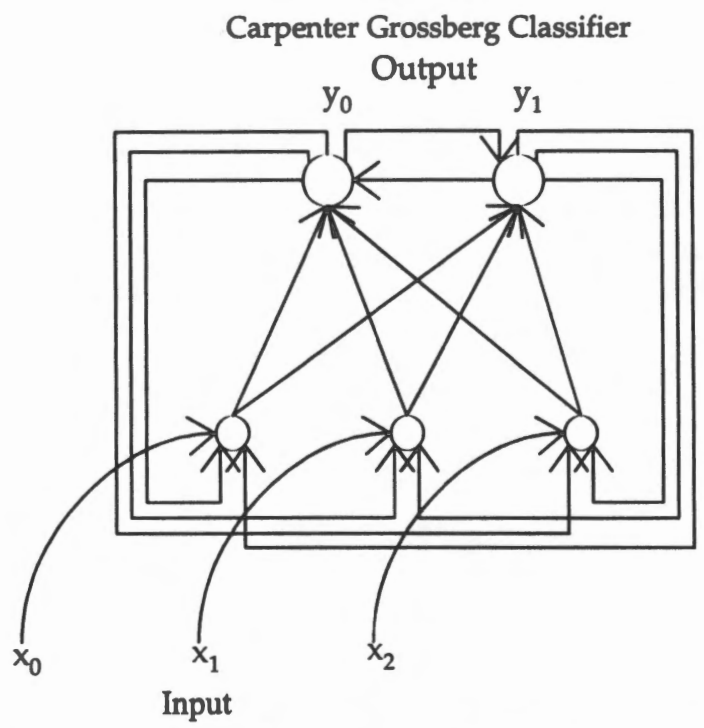

In the Carpenter Grossberg net, matching scores are computed using feedforward connections and the maximum value is enhanced using lateral inhibition among the output nodes. This net is structurally similar to the Hamming net (discussed below) but differs in that feedback connections are provided from the output nodes to the input nodes. This net is completely described using nonlinear differential equations, includes extensive feedback, and has been shown to be stable. Mechanisms are also provided to turn off that output node with the maximum value and to compare exemplars to the input for the threshold test required by the leader algorithm. The leader algorithm selects the first input as the exemplar for 
the first cluster. The next input is compared to the first cluster exemplar. It "follows the leader" and is clustered with the first if the distance to the first is less than a threshold. Otherwise, it is the exemplar for a new cluster. This process is repeated for all the following inputs. The number of clusters thus grows with time and depends on both the threshold and the distance metric used to compare inputs to cluster exemplars.

\section{Supervised Learning}

The following models, while fundamentally relying on the same neurobiological foundations as Hebb type learning, have an added dimension of "supervision." In supervised learning, it is assumed that for each instant of time when the input is applied the desired output is known through an exemplar. Supervised learning reduces to minimization of error in multidimensional weight space. Supervision is very important in models where convergence is presumed (or tests of convergence are of interest) or where a regional or macro policy influence may be considered relevant.

\section{Multilayer Perceptron}

The multilayer perceptron described below is a feedforward net used for optimization, classification, and prediction with one or more layers of nodes between the input and output nodes. As discussed above in the section on Hebb type learning, the ability of these systems to "learn" is based on the thesis that information is stored in connections. The added layers in this network allow for more connection, therefore, more complex classifications of inputs. A three-layer perceptron with one hidden layer, nine nodes in the input layer, and three nodes in the output layer is shown in Figure 5.

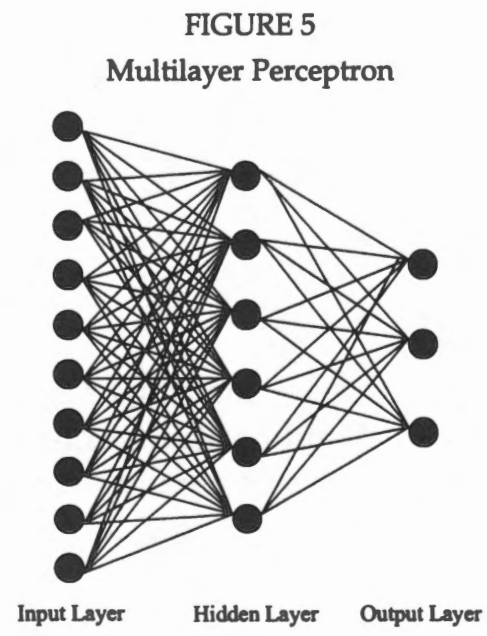

Rather than arranging the inputs in a three-by-three topographical grid, they are arranged in a linear fashion to demonstrate the number of weight connections generated by additional layers. 
The learning rule for this algorithm is given below.

Learning Algorithm:

1. Initialize weights $w_{i j}$ and offsets $\theta_{i j}$ to small random values.

2. Present the input $\left(X_{1}, X_{2}, \ldots, X_{9}\right)$ and the desired output 1 or 0.

3. Calculate the actual outputs based on

$$
y_{i}=f\left(\sum_{j=1}^{n} w_{i j} x_{j}-\theta\right),
$$

where $f$ is the sigmoid nonlinearity, given by

$$
f(x)=\frac{1}{1+e^{-x}},
$$

and $y_{i}$ is the output of node $i, x_{j}$ is the input from node $j$ of the previous layer, $w_{i j}$ is the weight from node $j$ to node $i$, and $\theta_{i}$ is the threshold for node $i$.

4. Update the weights according to the following rule:

$$
\Delta \mathbf{w}_{\mathrm{ij}}=\eta \delta_{\mathrm{i}} \mathbf{x}_{\mathrm{j}},
$$

where the error term $\delta_{i}$ in the update rule is defined as:

$$
\delta_{i}=y_{i}\left(1-y_{i}\right)\left(d_{i}-y_{i}\right),
$$

if node $i$ is the output node, or

$$
\delta_{i}=y_{i}\left(1-y_{i}\right) \sum \delta_{k} w_{i k} \text {, }
$$

if node $i$ is a hidden node, and $\eta$ is the learning rate parameter.

5 . Repeat by going to step 2 .

The process is repeated until the net stabilizes; that is, the weights cease to change appreciably as compared to some threshold. Here the "learning" or updating of the weights is based on the difference between the value at the output node and an exemplar value $d_{i}$. This error is then sent back and used to adjust the weights through the layers until the desired response occurs. In the neural net literature, this algorithmic process is referred to as "backpropogation."

\section{Hopfield Model}

The Hopfieid net also falls under the domain of supervised learning. There are many different versions of the Hopfield net. This model has evolved into an entire class of net architectures and has found wide applications to many optimization and classification problems in different fields. The model described here is one version of the original net (Hopfield 1982). A pictorial representation is given in Figure 6.

This net has $\mathrm{N}$ nodes containing hardlimiting nonlinearities and binary input and output that take values 1 or -1 . The output of each node is fed back to all other nodes via weights denoted by $t_{i j} \cdot x_{i}^{8}$ is element $i$ of the exemplar for class $\mathrm{s} ; \mu_{\mathrm{j}}(\mathrm{t})$ is the output of node $j$ at time $t$. 
FIGURE 6

Hopfield Model

Outputs

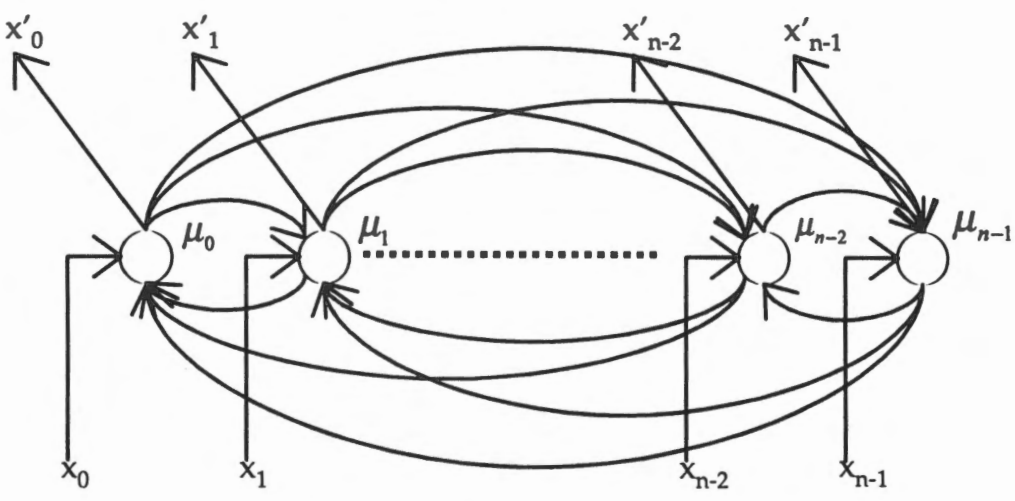

Inputs

The net algorithm works as follows:

1. Assign connection weights using

$$
\begin{aligned}
& w_{i j}=\sum_{s=0}^{M-1} x_{j}^{s} x_{i}^{s}, \quad i \neq j \\
& w_{i j}=0, i=j, 0 \leq(i, j) \leq M-1 .
\end{aligned}
$$

2. Initialize with the unknown input pattern

$$
\mu_{\mathrm{i}}(0)=\mathrm{x}_{\mathrm{i}}, 0 \leq \mathrm{i} \leq \mathrm{N}-1 \text {. }
$$

3. Iterate until convergence

$$
\mu_{j}(t+1)=f_{h}\left[\sum_{i=0}^{N-1} w_{i j} \mu_{i}(t)\right], 0 \leq j \leq M-1 .
$$

The function $f_{\mathrm{h}}$ is a hardlimiting nonlinearity that takes the value of 1 if it is greater than a threshold and -1 otherwise. The process is repeated until node outputs remain unchanged. The node output then represents the exemplar pattern that best matches the unknown input.

4. Repeat by going to step 2 .

This application of the Hopfield net is a classification problem, but it has been successfully applied to optimization problems as well as in the traveling salesman problem mentioned above.

\section{Boltzman Machines}

Boltzman machines (Figure 7) may be seen as an extension of Hopfield networks to include hidden units such as those in the multilayer perceptron. In 
Boltzman machines, probabilities of the states of the system are given by the Boltzman distribution of statistical mechanics and the learning rule is applicable to any stochastic network with symmetric connections. In its original form, the Boltzman learning algorithm is very slow because of the need for extensive averaging over stochastic variables, but a deterministic "mean field" version of the algorithm speeds up the algorithm considerably.

FIGURE 7

Boltzman Machine

(a)

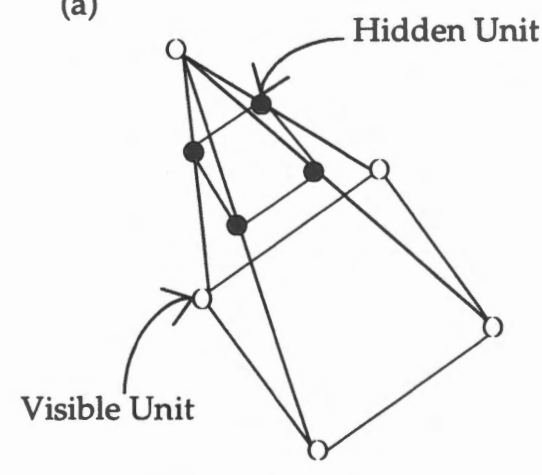

Output units

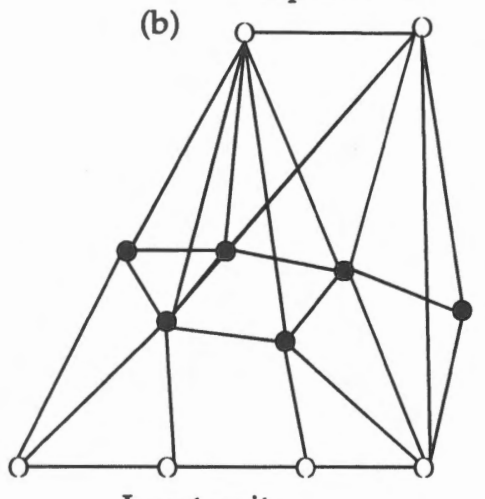

Input units

A Boltzman machine has the units divided into visible and hidden units. The visible units may be further subdivided into separate input and output units, the only restriction being that the weights between the units are symmetric, that is, $\mathbf{w}_{\mathrm{ij}}=\mathrm{w}_{\mathrm{jj}}$.

\section{Hamming Net}

The Hamming net is also an extension of the Hopfield net architecture. In the Hamming net, the weights and thresholds are first set in the lower subnet such that the matching scores generated by the outputs of the middle nodes are equal to $\mathrm{N}$ minus the Hamming distances to the exemplar patterns. The Hamming distance is the number of bits in the input that do not match the exemplar. The matching scores will range from 0 to the number of elements in the input $(\mathrm{N})$ and are highest for those nodes corresponding to classes with exemplars that best match the input. Thresholds and weights in the MAXNET subnet are fixed. All thresholds are set to 0 and weights from each node to its self are set to 1 . Weights between nodes are inhibitory with a value of $-\varepsilon$ where $\varepsilon<1 / \mathrm{M}$.

After weights and thresholds have been set, a binary pattern with $\mathrm{N}$ elements is presented at the bottom of the Hamming net. It must be presented long enough to allow the matching score outputs of the lower subnet to settle and initialize the output values of the MAXNET. The input is then removed and the MAXNET iterates until the output of only one node is positive. Classification is then complete and the selected class is that corresponding to the node with the positive output. The architecture of the Hamming net is represented in Figure 8. 
FIGURE 8

Hamming Net

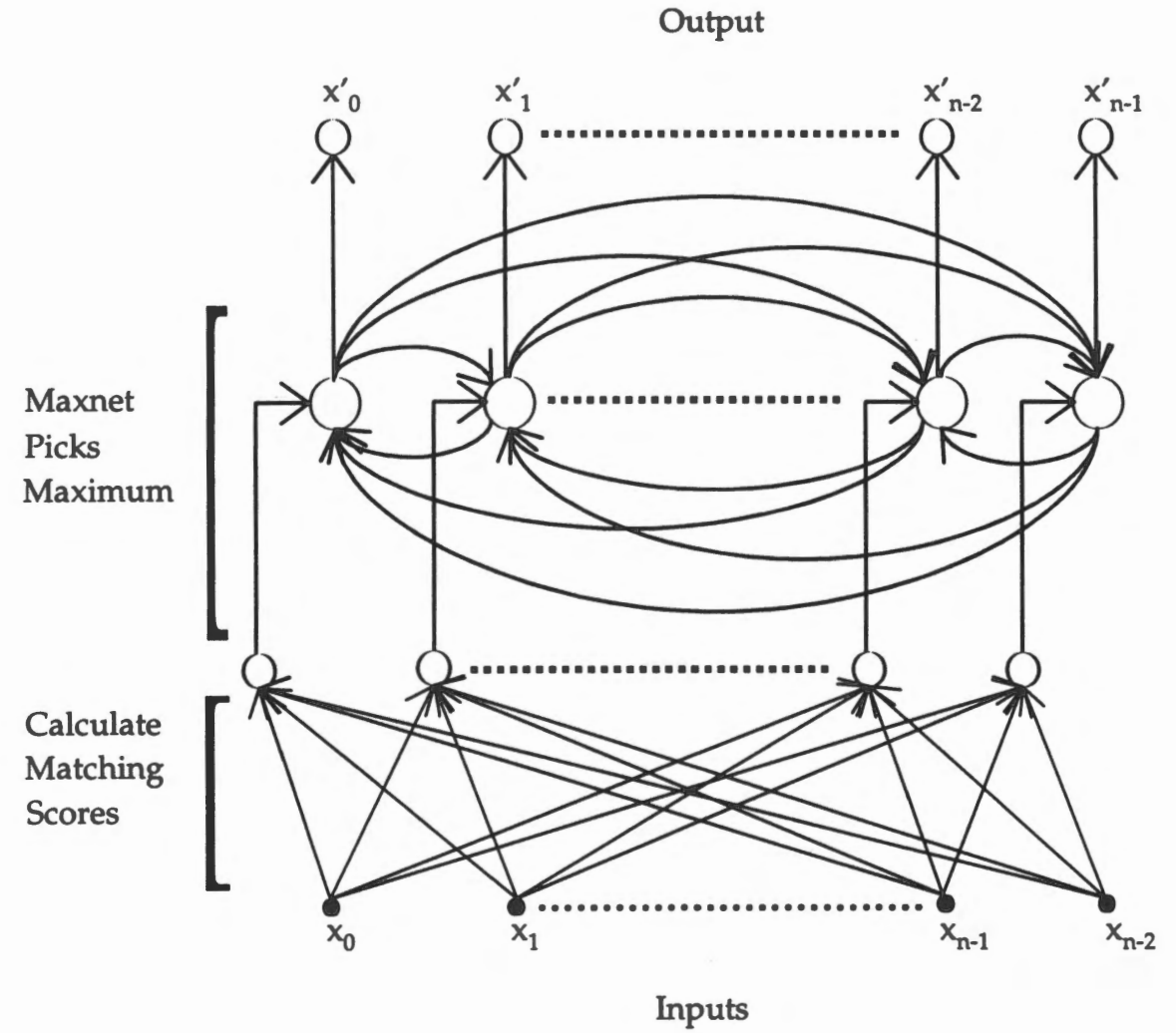

\section{CONCLUSION}

It has been demonstrated herein that the natural structure of these models matches well with the temporal-spatiality of regional science problems in general and with migration and firm location models in particular. The survey of the varied techniques above indicates that there is a tremendous opportunity for regional science approaches in economics to benefit from neural network modeling. The key element in unlocking the potential of these computational methods is sufficient interdisciplinary communication to specifically identify the relation between the structural architecture and functional decision rules inherent in these models, created mainly for computer science and engineering, to applications in regional science. In order to discover how the assumptions about economic behavior across geographic space relate to the apparently analogous structural assumptions in these various neural network models, the structure of each problem must be dissected and the theoretical assumptions made explicit to determine which models best represent particular regional economic phenomena. 


\section{REFERENCES}

Alonso, W. "A Reformation of Classical Location Theory and its Relation to Rent Theory." In G.J. Karaska and D.F. Bramhall (eds.) Locational Analysis for Manufacturing, 35-63. Cambridge: MIT Press, 1969.

Barloon, M. "The Interrelationship of the Changing Structure of American Transportation and Changes in Industrial Location." In G.J. Karaska and D.F. Bramhall (eds.) Locational Analysis for Manufacturing, 97-107. Cambridge: MIT Press, 1969.

Carpenter, G.A., and S. Grossberg. "Neural Dynamics of Category Learning and Recognition: Attention, Memory Consolidation, and Amnesia." In J. Davis, R. Newburgh, and E. Wegman (eds.) Brain Structure, Learning, and Memory. AAAS Selected Symposium no. 105. Boulder, Colo.: Westview Press, 1988.

Chinitz, B. "The Effect of Transportation Forms on Regional Economic Growth." In G.J. Karaska and D.F. Bramhall (eds.) Locational Analysis for Manufacturing, 83-96. Cambridge: MIT Press, 1969.

Greenwood, M. "An Analysis of the Determinants of Geographic Labor Mobility in the United States." Review of Economics and Statistics 51 (2) (1969), 189-194.

Hebb, D.O. The Organization of Behavior: A Neuropsychological Theory. New York: John Wiley, 1949.

Hertz, J., A. Krogh, and R.G. Palmer. Introduction to the Theory of Neural Computation. Lecture Notes, Vol. 1. Reading, Mass.: Addison-Wesley Publishing Co., 1991.

Herzog, H., and A. Schlottman. "Worker Displacement and Job-Search: A Regional Analysis of Structural Impediments to Reemployment." Journal of Regional Science 35 (4) (1995), 553-577.

"Moving Back vs. Moving On: The Concept of Home in the Decision to Remigrate." Journal of Regional Science 22 (1) (1982), 73-82.

"Labor Force Migration and Allocative Efficiency in the United States:

The Roles of Information and Psychic Costs." Economic Inquiry 19 (3) (1981), 43-56.

Herzog, H., T.P. Boehm, and A. Schlottman. "Migration as Spatial Job-Search: A Survey of Empirical Findings." Regional Studies 27 (4) (1993), 327-340.

Hoover, E.M. The Location of Economic Activity. New York: McGraw Hill, 1948.

Hopfield, J.J. "Neurons with Graded Response have Collective Computational Properties Like those of Two State Neurons." Proceedings of the National Academy of Science USA 81 (1984), 3088-3092.

. "Neural Networks in Physical Systems with Emergent Collective Computational Abilities." Proceedings of the National Academy of Science USA 79 (1982), 2554-2558.

Hopfield, J.J., and D.W. Tank. "Neural Computation of Decisions in Optimization Problems." Biological Cybernetics 52 (1985), 141-154.

Hotelling, H. "Stability in Competition." In R.D. Dean, W.H. Leahy, and D.L. McKee (eds.) Spatial Economic Theory, 103-118. New York: The Free Press, 1970. 
Isard, W. Location and Space-Economy. New York: Published jointly by the Technical Press of MIT and Wiley, 1956.

Kau, J., and C.F. Sirmans. "The Influence of Information Costs and Uncertainty on Migration: A Comparison of Migration Types." Journal of Regional Science 17 (1) (1977), 89-96.

Levy, M., and W. Wadycki. "The Influence of Family and Friends on Geographic Labor Mobility: An International Comparison." Review of Economics and Statistics 55 (2) (1973), 198-203.

Moses, L. "Location and the Theory of Production." In R.D. Dean, W.H. Leahy, and D.L. McKee (eds.) Spatial Economic Theory, 59-71. New York: The Free Press, 1970.

Palander, T. Beitrage zur Standortstheorie. Uppsala, Sweden: Almquist Och Wiksells Botryckeri-a.-b., 1935.

Phoha, V., and W.J.B. Oldham. Corrections to "Image Recovery and Segmentation using Competitive Learning in a Layered Network." IEEE Transactions on Neural Networks 7 (6) (1996a), 1547-1548. - "Image Recovery and Segmentation using Competitive Learning in a Layered Network." IEEE Transactions on Neural Networks 7 (4) (1996b), 843-856.

Smith, D. Industrial Location: An Economic Geographical Analysis. New York: Wiley, 1971.

Weber, A. Theory of the Location of Industry. Chicago: University of Chicago Press, 1928.

Zurada, J.M. Introduction to Artificial Neural Systems. St. Paul, Minn.: West Publishing Co., 1992. 